Alexandre Barreto ALMEIDA ${ }^{1}$

Claudia Maria BERTAN ${ }^{1}$

Luis Augusto Ferreira

ROSSA $^{1}$

Pércio dos Santos GASPAR

Mario BINELLI ${ }^{1}$

Ed Hoffmann MADUREIRA

Correspondência para:

ED HOFFMANN MADUREIRA

Centro de Biotecnologia em Reprodução Animal

Faculdade de Medicina Veterinária e Zootecnia

Universidade de São Paulo

Av. Duque de Caxias Norte, 225

13630-000 - Pirassununga -SP

madureh@usp.br

Recebido para publicação: 15/07/2005 Aprovado para publicação: 23/08/2005

\title{
Avaliação da reutilização de implantes auriculares contendo norgestomet associados ao valerato ou ao benzoato de estradiol em vacas nelore inseminadas em tempo fixo
}

1 - Departamento de Reprodução Animal da Faculdade de Medicina Veterinária e Zootecnia da Universidade de São Paulo, Pirassununga - SP

\section{Resumo}

Em fêmeas bovinas a utilização da técnica de inseminação artificial em tempo fixo (IATF) é possibilitada pelo emprego de fármacos que tem como objetivos sincronizar a emergência das ondas foliculares, os estros e a ovulação. Em rebanhos comerciais, o custo de tais fármacos deve estabelecer uma vantajosa relação com os benefícios. $\mathrm{O}$ presente estudo, objetivou comparar as taxas de prenhez em vacas Nelore (Bos taurus indicus) inseminadas em tempo fixo, tratadas com implantes auriculares contendo $3 \mathrm{mg}$ de norgestomet $\left(\right.$ Crestar $\left.^{\circledR}\right)$, novos (IN) ou utilizados uma vez (IR; reutilizados), associados a administração de norgestomet (NG) e valerato de estradiol (VE) ou progesterona $\left(\mathrm{P}_{4}\right)$ e benzoato de estradiol (BE). Vacas Nelore PO ( $\mathrm{n}=241)$, amamentando, com o bezerro ao pé, receberam um dos quatro tratamentos: Crestar $^{\circledR}$ novo durante 10 dias associado a administração de $3 \mathrm{mg}$ de $\mathrm{NG}$ e $5 \mathrm{mg}$ de $\mathrm{VE}$ (grupo IN/NG+VE; $\mathrm{n}=61)$; Crestar $^{\circledR}$ novo inserido durante 8 dias associado a $50 \mathrm{mg}$ de $\mathrm{P}_{4}$ e $2 \mathrm{mg}$ de $\mathrm{BE}$ (grupo IN $/ \mathrm{P}_{4}+\mathrm{BE}$; $\mathrm{n}=61$ ); Crestar $^{\circledR}$ reutilizado inserido durante 10 dias associado a administração de $3 \mathrm{mg}$ de $\mathrm{NG}$ e $5 \mathrm{mg}$ de

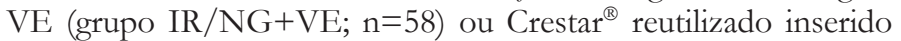
durante 8 dias associado a e $50 \mathrm{mg}$ de $\mathrm{P}_{4}$ e $2 \mathrm{mg}$ de $\mathrm{BE}$ (grupo IR/ $\left.\mathrm{P}_{4}+\mathrm{BE} ; \mathrm{n}=61\right)$. No dia da remoção dos implantes os animais receberam 7,5mg de Luprostiol e 24 horas após a remoção $1 \mathrm{mg}$ de BE. A IATF foi realizada 54 a 56 horas após a retirada dos implantes. Após a IATF, as vacas tiveram os estros observados durante um período de 49 dias e em estro foram reinseminadas 12 horas após a observação. O diagnóstico de gestação foi realizado por ultra-sonografia 35 dias após a IATF e após o final da estação de monta. Foram avaliadas as taxas de prenhez na IATF (TP IATF) e no final da estação de monta (TP EM). Não houve interação entre as características dos implantes (novos e reutilizados) e os tratamentos administrados no dia da inserção do implante $\left(\mathrm{NG}+\mathrm{VE}\right.$ e $\left.\mathrm{P}_{4}+\mathrm{BE}\right)$. A utilização do $\mathrm{CIDR}^{\circledR}$ novo ou reutilizado não teve efeito nas TP IATF (48,3\% vs $48,7 \%$ ) e nas TP EM (85,2 vs 86,5\%). Os tratamentos com NG+VE e $\mathrm{P}_{4}+\mathrm{BE}$ não tiveram efeito nas TP IATF (49,5 vs 47,5\%) e TP EM $(86,5$ vs $85,2 \%)$. Houve efeito do número de parições (primíparas e multíparas) nas TP IATF ( $35 \%$ vs. $52,7 \%$; P<0,01) e nas TP EM (71,9\% vs. 90,2\%; $\mathrm{P}<0,01)$. Conclui-se que implantes de norgestomet novos e reutilizados, quando associados ao $\mathrm{NG}+\mathrm{VE}$ ou $\mathrm{P}_{4}+\mathrm{BE}$ promovem taxas de prenhez bastante satisfatórias em vacas Nelore e que melhores taxas são obtidas em fêmeas multíparas.
Palavras-chave: Progestágenos. Reutilização.

Valerato de estradiol. Benzoato de estradiol. Bovinos. 


\section{Introdução}

Em fêmeas bovinas a utilização da técnica de inseminação artificial (IA) pode ser facilitada pelo emprego de protocolos que promovam a sincronização da emergência das ondas foliculares, dos estros e da ovulação. O emprego de tais protocolos, dispensa a observação de estros e possibilita a realização da inseminação artificial em tempo fixo (IATF). Entretanto, os fármacos utilizados para promover tais eventos fisiológicos em programas de IATF devem oferecer uma vantajosa relação entre custos e benefícios.

Nos protocolos de IATF freqüentemente os progestágenos são associados à esteres de estradiol ${ }^{1,2}$ e a prostaglandina PGF2 $\alpha$ (PGF2 $\alpha$ ). Dentre os progestágenos, o $17 \alpha$-acetoxi-11 $\beta$-metil-19norpreg-4-en-3,30diona (norgestomet) impregnado em implantes auriculares, tem sido amplamente utilizado na sincronização dos estros em fêmeas bovinas. A liberação diária de norgestomet pelo uso de tais implantes, resulta em concentrações séricas de progesterona $\left(\mathrm{P}_{4}\right)$ que impossibilitam o pico pré-ovulatório de LH e a ocorrência de estros durante o tratamento. Mihm ${ }^{3}$ relata que tratamentos com progestágenos no período pós-parto induzem a retomada da ciclicidade. Garcia-Winder et $\mathrm{al}^{4}$ verificaram que vacas de corte no pós parto tratadas com norgestomet apresentaram aumento no diâmetro folicular e na atividade estrogênica. Entretanto, em fêmeas tratadas exclusivamente com progestágenos, verificou-se a ocorrência de folículos dominantes persistentes ${ }^{5}$. Considerando tal ocorrência, a associação de progestágenos ao ésteres de estradiol tem sido freqüentemente utilizada em protocolos que objetivam a sincronização de ondas foliculares e dos estros. A associação de tais fármacos também têm sido empregada no tratamento de vacas em anestro durante o período pósparto ${ }^{6}$.

Barufi $^{7}$ e et al ${ }^{8}$ verificaram que o uso de implantes auriculares contendo norgestomet, associado a administração de ésteres de estradiol no dia de inserção do implante, proporcionou taxas de prenhez de $45 \%$ em fêmeas de corte submetidas a IATF, quando os tratamentos foram iniciados a partir de 49 dias após o parto. Bo et $\mathrm{al}^{9}$ observaram que a administração de $5 \mathrm{mg}$ de valerato de estradiol (VE) resultou na inibição do crescimento do folículo dominante e na emergência de uma nova onda folicular 6,3 dias após o tratamento. Moreno et $\mathrm{al}^{10}$ administraram $2 \mathrm{mg}$ de benzoato de estradiol (BE) associado a $50 \mathrm{mg}$ de $\mathrm{P}_{4}$ e verificaram a emergência de uma nova onda folicular após 4,1 dias). O BE é uma forma esterificada do estradiol na posição $\mathrm{C}_{3} \mathrm{OH}$ (VYNCKIER et $\mathrm{al}^{11}$. O VE é um éster do $17 \beta$-estradiol, modificado na posição 17 , particularidade que lhe confere uma meiavida mais longa quando comparado ao $\mathrm{BE}$ WILLIAMS; STANCEL ${ }^{12}$. A administração de prostaglandina F2 $\alpha$ (PGF2 $\alpha$ ), 24 a 48 horas antes da retirada do implante, é recomendada com o objetivo de favorecer a sincronização dos estros ${ }^{13,14}$.

Madureira ${ }^{15}$ observaram que ao término do tratamento com progestágenos a população de folículos pré-ovulatórios foi bastante homogênea, condição que favorece a sincronização da ovulação. De fato, Colazo et al. ${ }^{16}$ e Martinez et al. ${ }^{17}$ verificaram que doses de 0,7 a $1 \mathrm{mg}$ de BE, 24 horas após a remoção de implantes de progestágenos, induziram a um pico pré-ovulatório de $\mathrm{LH}$ e a uma alta taxa de sincronização das ovulações.

Kesler et $\mathrm{al}^{18}$ e Machado e Kesler ${ }^{19}$ verificaram que doses diárias de 137 a $138 \mathrm{mg}$ de norgestomet foram requeridas para suprimir a manifestação de estro em todas as fêmeas tratadas. Kesler et $\mathrm{al}^{18}$ relataram que implantes auriculares silásticos, contendo 6 a $8 \mathrm{mg}$ de norgestomet, inseridos em vacas de corte durante 16 dias, tiveram a concentração de norgestomet reduzida para 3,04 e 3,76mg ao término do tratamento, ou seja, aproximadamente $50 \%$ da concentração inicial. Com base nesses dados, pressupõe-se que em implantes auriculares 
contendo $3 \mathrm{mg}$ de norgestomet utilizados durante 8 dias teriam a concentração inicial de norgestomet reduzida em $25 \%$ ao término do tratamento. Considerando que $138 \mathrm{mg}$ diárias de norgestomet são suficientes para suprimir o estro em vacas de corte, implantes auriculares contendo $3 \mathrm{mg}$ de norgestomet poderiam ser utilizados uma segunda vez (reutilizados). A possibilidade de reutilização constitui uma interessante alternativa na redução de custo dos protocolos que utilizam tais implantes. $\mathrm{O}$ objetivo desse estudo foi comparar as taxas de prenhez utilizando IATF em vacas da raça Nelore (Bos taurus indicus), lactantes, com bezerro ao pé, tratadas com implantes auriculares, novos e reutilizados, contendo norgestomet, associados a administração de norgestomet e VE ou a $\mathrm{P}_{4}$ e $\mathrm{BE}$ no dia da inserção do implante, em vacas injetadas com PGF2 $\alpha 24$ horas antes da retirada do implante e BE 24 horas após a retirada do mesmo. A hipótese do presente trabalho é que não há diferença nas taxas de prenhez entre implantes auriculares novos e reutilizados e que em vacas tratadas com $\mathrm{P}_{4}$ e BE no dia da colocação do implante auricular as taxas de prenhez são maiores do que em fêmeas tratadas com norgestomet e VE.

\section{Materiais e Métodos}

\section{Local do Experimento e Animais}

O experimento foi realizado na Fazenda Guanabara, pertencente a Agropecuária J. Galera, localizada no município de Itarumã, no estado de Goiás. Foram utilizadas 241 vacas Nelore PO (Bos taurus Indicus), de idade variável, lactantes e com bezerro ao pé. O experimento foi realizado em duas repetições. A primeira repetição foi realizada com 137 vacas em dezembro/2001 e a segunda com 104 vacas em março/2002. Durante a execução do experimento os animais foram mantidos em piquetes com água à disposição. A alimentação foi baseada no pastejo (Brachiaria brizanta var marandu) complementada com suplementação mineral.

\section{Delineamento Experimental}

As vacas foram distribuídas em quatro grupos de maneira eqüitativa, considerando-se: número de parições (primíparas ou multíparas), período pós parto, peso, condição corporal e condição ovariana. A média do período pós parto foi de $118 \pm 70,8$ e $77 \pm 28,1$ dias na primeira e segunda repetição, respectivamente. Considerando as 241 vacas utilizadas, 35\% eram primíparas e $65 \%$ multíparas. A média de peso foi de $455 \pm 47,9$ na primeira repetição e $479 \pm 57,8 \mathrm{Kg}$ na segunda. A condição corporal média foi de 4,8 $\pm 0,8$ e $4,7 \pm 0,9$ na primeira e segunda repetição, respectivamente. A condição ovariana foi avaliada por palpação retal e classificada em três condições: vacas cíclicas com folículos $\geq 10 \mathrm{~mm}$ e corpo lúteo presente (condição $1)$; presença de folículos dominantes $\geq$ $8,5 \mathrm{~mm}$ anovulatórios e corpo lúteo ausente (condição 2) e presença de ovários pequenos, duros e lisos, com folículos que não atingiam a fase de divergência (condição 3). A média da condição ovariana foi de $1,2 \pm 0,5$ e 1,3 $\pm 0,5$ na primeira e segunda repetição, respectivamente. A obtenção dos implantes de Crestar $^{\circledR}$ (Intervet International B.V. Boxmeer - Holanda) reutilizados consistiu na retirada do implante após o mesmo ser utilizado durante 10 dias, lavagem com água destilada e dezionizada, secagem em papel absorvente e conservação a $4^{\circ} \mathrm{C}$ por aproximadamente quatro meses. No dia da inserção dos implantes auriculares as vacas receberam um dos quatro tratamentos: Crestar $^{\circledR}$ novo que permaneceu inserido durante 10 dias associado a administração de $3 \mathrm{mg}$ de norgestomet (Intervet International B.V. Boxmeer - Holanda) via intramuscular (IM) e 5mg de VE (Intervet International B.V. Boxmeer - Holanda) via IM (grupo IN/NG+VE; n=61); Crestar $^{\circledR}$ novo inserido durante 8 dias associado $50 \mathrm{mg}$ de $\mathrm{P}_{4}$ (Index Farmacêutica - Brasil) e a $2 \mathrm{mg}$ de BE (Index Farmacêutica - Brasil), ambos administrados via IM (grupo IN/ $\mathrm{P}_{4}+\mathrm{BE}$; 
$\mathrm{n}=61)$; Crestar $^{\circledR}$ reutilizado inserido durante 10 dias associado a administração de $3 \mathrm{mg}$ de norgestomet e $5 \mathrm{mg}$ de $\mathrm{VE}$, ambos via IM (grupo IR/NG+VE; $n=58$ ) ou Crestar $^{\circledR}$ reutilizado inserido durante 8 dias associado $50 \mathrm{mg}$ de $\mathrm{P}_{4}$ (Index Farmacêutica - Brasil) e a $2 \mathrm{mg}$ de BE (Index Farmacêutica - Brasil), ambos administrados via IM (grupo IR/ $\left.\mathrm{P}_{4}+\mathrm{BE} ; \mathrm{n}=61\right)$. No dia da remoção dos implantes todos os animais receberam $7,5 \mathrm{mg}$ de Luprostiol (Prosolvin ${ }^{\circledR}$ - Intervet International B.V. Boxmeer - Holanda) e 24 horas após a remoção dos implantes receberam $1 \mathrm{mg}$ de BE (Index Farmacêutica, São Paulo, Brasil) via IM. A IATF foi realizada 54 a 56 horas após a retirada dos implantes. O dia da IATF foi considerado o primeiro dia da estação de monta. Após a IATF dois rufiões foram introduzidos no lote de vacas para auxiliar na detecção de estros, realizada duas vezes ao dia, pela manhã e à tarde. Vacas detectadas em estro após a realização da IATF foram inseminadas 12 horas após, durante um período de estação de monta de 49 dias. Para a IATF utilizou-se sêmen congelado de 20 touros da raça Nelore com concentração, características físicas e morfológicas dentro dos padrões recomendados pelo Ministério da Agricultura, Pecuária e Abastecimento (MAPA). Os touros foram divididos eqüitativamente entre os diferentes grupos de tratamento. $O$ diagnóstico de gestação foi realizado por ultra-sonografia (Aloka SSD500; transdutor linear de 5MhZ) 35 dias após a IATF. Após o final da estação de monta realizou-se novo exame ultrasonográfico.

\section{Análise Estatística}

O delineamento experimental foi o de blocos casualizados generalizados, em um arranjo fatorial $2 \times 2$ (Implante Crestar ${ }^{\circledR}$ novo (IN) ou reutilizado (IR) $x \mathrm{NG}+\mathrm{VE}$ ou $\left.\mathrm{P}_{4}+\mathrm{BE}\right)$. As análise foram realizadas pelo programa Statistical Analysis System ${ }^{23}$ (SAS, Institute Inc., 1985). Os dados foram analisados por ANOVA, utilizando-se o proc GLM do programa SAS (SAS Institute,
1988). No modelo, as variáveis dependentes foram: taxa de prenhez na IATF (TP IATF) e taxa de prenhez no final da estação de monta (TP EM). As variáveis independentes foram: característica do implante (novo ou reutilizado), tratamentos administrados no dia da inserção do implante (NG+VE ou $\mathrm{P}_{4}+\mathrm{BE}$ ), número de parições (primípara ou multípara), período pós parto, condição corporal, condição ovariana e interações apresentadas na tabela 1 .

Também foram representadas a porcentagem de vacas vazias ao longo dos dias da estação de monta, baseando-se nas datas da realização das inseminações, segundo a característica do implante (novo ou reutilizado) e o tratamento administrado no dia da inserção do implante $(\mathrm{NG}+\mathrm{VE}$ ou $\left.\mathrm{P}_{4}+\mathrm{BE}\right)$.

\section{Resultados e discussões}

No presente estudo, durante o período em que as vacas foram tratadas com os implantes auriculares, novos e reutilizados, não houve a manifestação de estros, o que sugere que os implantes reutilizados liberam no mínimo a quantidade necessária para suprimir a ocorrência de estros, que segundo Kesler et al ${ }^{18}$ é de 137 a $138 \mathrm{mg}$ ao dia em vacas Bos taurus taurus. Especula-se que os implantes comercialmente disponíveis contendo $3 \mathrm{mg}$ de norgestomet, sejam capazes de liberar a quantidade mínima do progestágeno suficiente para suprimir os estros por pelo menos 20 dias. Ainda devese considerar que em vacas Nelore amamentando a dose mínima para suprimir o estro é menor do que a necessária para provocar o mesmo efeito em vacas Bos taurus taurus.

Conforme representado na tabela 1 , não houve interação entre a característica do implante (novo ou reutilizado) e o tratamento administrado no dia da inserção do implante $\left(\mathrm{NG}+\mathrm{VE}\right.$ ou $\left.\mathrm{P}_{4}+\mathrm{BE}\right)$, portanto os resultados serão discutidos nos efeitos principais. As TP IATF foram de 48,3\% e $48,7 \%$ quando utilizou-se implantes novos e 
reutilizados, respectivamente. As TP EM foi de $85,2 \%$ para implantes novos e $86,5 \%$ para reutilizados. Na figura 1 estão representadas a porcentagem de vacas vazias ao longo dos dias da estação de monta quando foram utilizados implantes novos e reutilizados. Verificou-se que as taxas de prenhez (TP IATF e TP EM) foram igualmente satisfatórias para implantes novos e reutilizados, pois $48 \%$ das vacas conceberam na inseminação realizada no primeiro dia de estação de monta. Barufi ${ }^{7}$ e Barufi $^{8}$ comparou a eficiência do Crestar ${ }^{\circledR}$ novo e do dispositivo intravaginal contendo progesterona (CIDR) novo e reutilizado em fêmeas de corte e verificou após a IATF taxas de prenhez de $52,7 \%, 28,8 \%$ e $38,7 \%$, respectivamente, e verificou maiores taxas $(\mathrm{P}<0,05)$ quando o Crestar $^{\circledR}$ foi utilizado.

As TP IATF representadas na tabela 1, foram de 49,5\% e 47,5\% para os tratamentos com $\mathrm{NG}+\mathrm{VE}$ e $\mathrm{P}_{4}+\mathrm{BE}$, respectivamente. A TP EM foi de 86,5\% em vacas tratadas com NG+VE e de 85,2\% para as tratadas com $\mathrm{P}_{4}+\mathrm{BE}$. Na figura 2 estão representadas as porcentagens de vacas vazias ao longo dos dias da estação de monta quando tratadas com $\mathrm{NG}+\mathrm{VE}$ e $\mathrm{P}_{4}+\mathrm{BE}$. No presente experimento, esperava-se que o tratamento $\mathrm{NG}+\mathrm{VE}$ resultasse em uma menor TF IATF quando fosse comparado ao $\mathrm{P}_{4}+\mathrm{BE}$. $\mathrm{A}$ administração de ésteres de estradiol no dia da inserção do implante teve como objetivo promover a atresia do folículo dominante e promover a emergência de uma nova onda folicular. No presente trabalho, foram utilizados dois diferentes tipos de ésteres de estradiol, o VE e o BE. Quando utilizou-se o VE, o período de permanência do implante foi de 10 dias, enquanto para o BE foi de apenas 8 dias. Tal estratégia possibilitou que, no momento da retirada dos implantes, os folículos da onda folicular apresentassem um maior grau de sincronização, pois estariam entre o $4^{\circ} \mathrm{e}$ $5^{\circ}$ dias após a emergência, independentemente do éster de estradiol empregado. De fato, as TP IATF e TP EM foram muito semelhantes para o VE e BE.
As TP IATF obtidas no presente estudo, podem ser consideradas muito boas e são semelhantes às obtidas por outros autores ${ }^{21,22}$. Ressalta-se que a grande vantagem do emprego de tais protocolos é a ocorrência de uma taxa de prenhez de $48,4 \%$ no primeiro dia da estação de monta. Outra diferença básica entre os efeitos do VE e do $\mathrm{BE}$ diz respeito à eficiência em induzir a luteólise. O BE é menos eficiente que o VE para promover a luteólise ${ }^{21}$. Dessa maneira, preconizou-se a aplicação de PGF2a no momento da retirada dos implantes, com o objetivo de assegurar a luteólise para todos os tratamentos.

Segundo Mcguire, Larson e Kiracofe $^{23}$ em vacas tratadas com norgestomet e VE pode ocorrer a manifestação comportamental de estro independente da atividade ovariana. Entre as possíveis explicações para este fato, os autores relacionaram a sensibilização dos animais ao estrógeno extraovariano e a presença de metabólitos com atividade estrogênica residual decorrente do tratamento. De fato, a indução dos estros na ausência da ovulação foi mais comumente observada na associação do norgestomet com o VE. Entretanto, no presente experimento, a manifestação de estros seguida pela ausência de ovulações provavelmente não tenha sido freqüente, visto que as taxas de prenhez foram elevadas.

Burke et $\mathrm{al}^{24}$ administraram $1 \mathrm{mg}$ de $\mathrm{BE}$ via $\mathrm{IM}$ e constaram um rápido aumento na concentração plasmática de estradiol, a concentração máxima ocorreu na hora 4 $(12,6 \pm 1,6 \mathrm{pg} / \mathrm{ml})$ e permaneceu elevada $(12 \mathrm{pg} / \mathrm{ml})$ até 24 após administração. Vynckier et al $^{11}$ também relatou um rápido aumento na concentração plasmática de estradiol após a aplicação de $10 \mathrm{mg}$ de BE por via IM. De fato, Colazo et al ${ }^{16} \mathrm{e}$ Martinez et $\mathrm{al}^{17}$ verificaram que doses de $0,7 \mathrm{a} 1 \mathrm{mg}$ de BE, 24 horas após a remoção de implantes de progestágenos, induziram a um pico préovulatório de LH e a uma alta taxa de sincronização das ovulações. No presente estudo, considerando as taxas de prenhez obtidas após a IATF sugere-se que o BE foi 


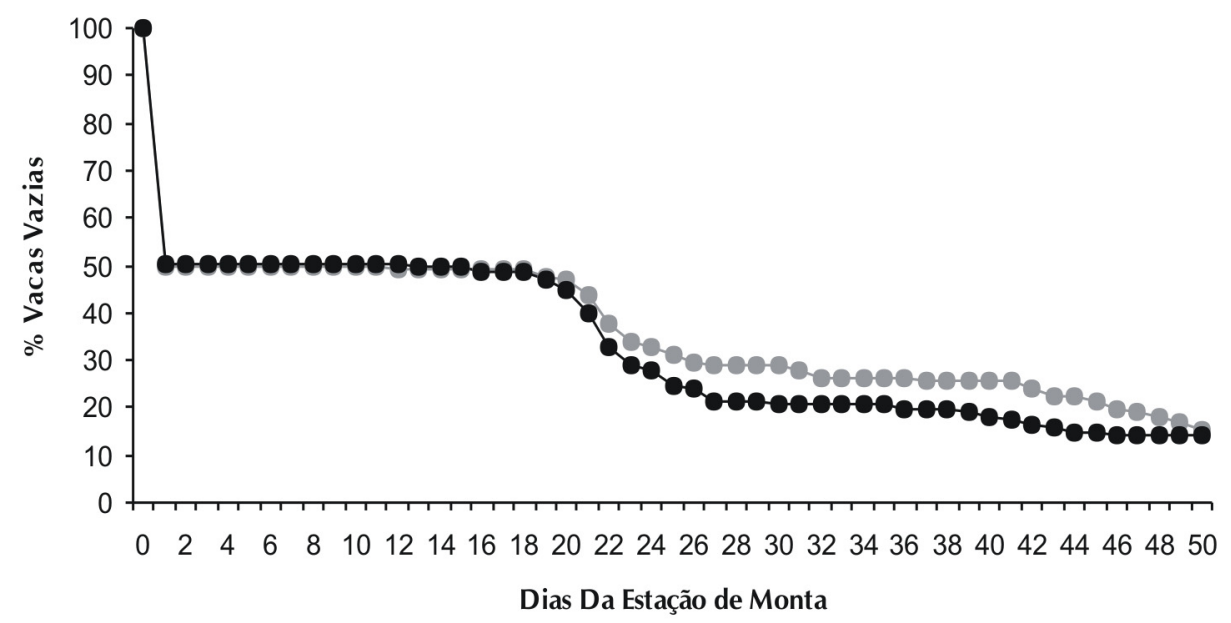

Figura 1 - Porcentagem de fêmeas vazias ao longo dos diferentes dias da estação de monta, em experimento realizado com vacas Nelore, amamentando, tratadas com implantes silásticos contendo 3 mg de norgestomet $\left(\right.$ Crestar $\left.^{\circledR}\right)$ novos $(\bullet ; n=112)$ ou reutilizados $(\bullet ; n=119)$ associados a administração de norgestomet e valerato de estradiol ou progesterona e benzoato de estradiol no dia da colocação dos implantes em vacas que receberam 7,5mg de Luprostiol na remoção dos implantes e 24 horas após a remoção $1 \mathrm{mg}$ de benzoato de estradiol e foram submetidas a IATF 54 a 56 horas após a retirada dos implantes. As vacas que tiveram os estros observados durante 49 dias após a IATF foram novamente inseminadas.

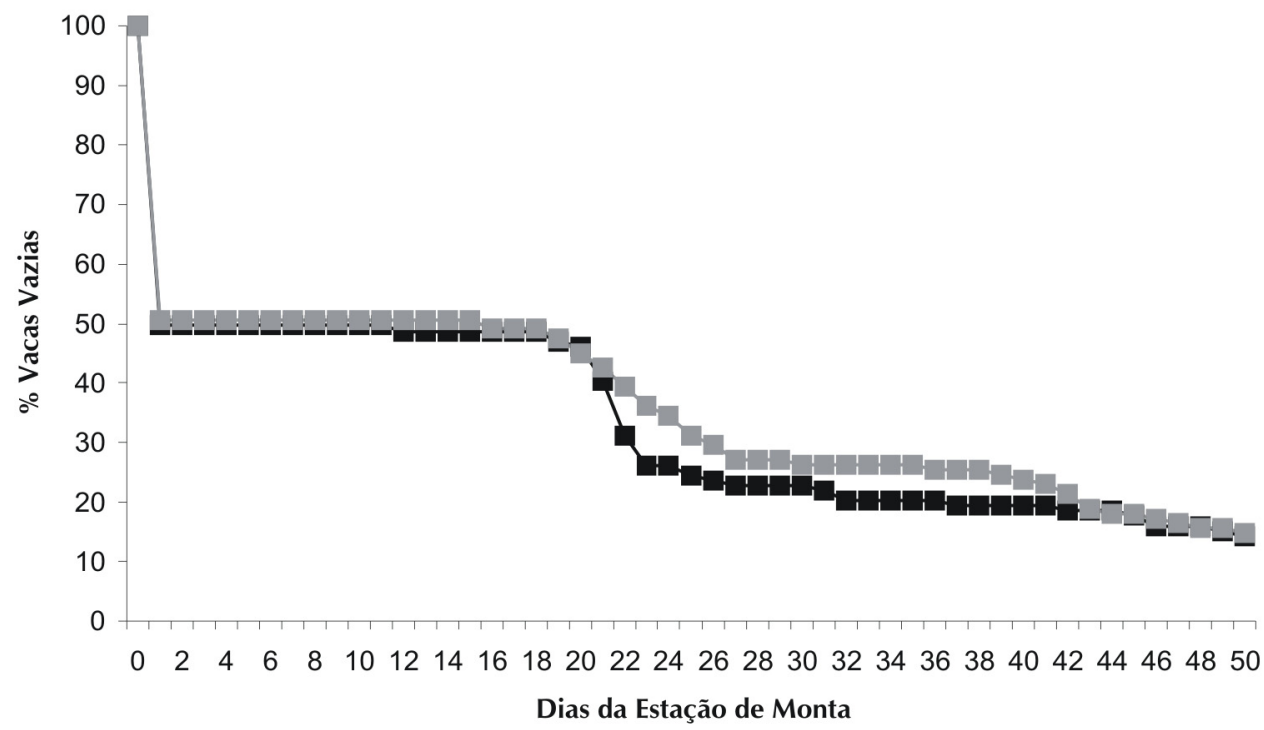

Figura 2 - Porcentagem de fêmeas vazias ao longo dos diferentes dias da estação demonta, em experimento realizado com vacas Nelore, amamentando, tratadas com implantes silásticos contendo $3 \mathrm{mg}$ de norgestomet (Crestar ${ }^{\circledR}$ ) novos ou reutilizados associados a administração de norgestomet e valerato de estradiol $(\mathbf{\square} ; \mathrm{n}=119)$ ou progesterona e benzoato de estradiol $(\mathbf{\square} ; \mathrm{n}=112)$ no dia da colocação dos implantes em vacas que receberam 7,5mg de Luprostiol na remoção dos implantes e 24 horas após a remoção $1 \mathrm{mg}$ de benzoato de estradiol e foram submetidas a IATF 54 a 56 horas após a retirada dos implantes. As vacas que tiveram os estros observados durante 49 dias após a IATF foram novamente inseminadas 


\section{INTERAÇÕES}

\begin{tabular}{|c|c|c|c|}
\hline $\begin{array}{l}\text { Característica do } \\
\text { implante }\end{array}$ & $\begin{array}{l}\text { Tratamento administrado } \\
\text { no dia da colocação do } \\
\text { implante }\end{array}$ & TP IATF $(\%)$ & TP EM (\%) \\
\hline Novos & $N G+V E(n=61)$ & 49,1 & 85,2 \\
\hline Novos & $P_{4}+B E(n=61)$ & 47,5 & 85,2 \\
\hline Reutilizados & $N G+V E(n=58)$ & 50,0 & 87,9 \\
\hline Reutilizados & $P_{4}+B E(n=61)$ & 47,5 & 85,2 \\
\hline \multicolumn{4}{|c|}{ EFEITOS PRINCIPAIS } \\
\hline $\begin{array}{l}\text { Característica do } \\
\text { implante }\end{array}$ & $\begin{array}{l}\text { Tratamento administrado } \\
\text { no dia da colocaçãa do } \\
\text { implante }\end{array}$ & TP IATF (\%) & $\operatorname{TP}$ EM (\%) \\
\hline \multirow[t]{2}{*}{ Novos $(n=122)$} & & 48,3 & 85,2 \\
\hline & & 48,7 & 86,5 \\
\hline \multirow{4}{*}{$\begin{array}{l}\text { Reutilizados } \\
\qquad(\mathrm{n}=119)\end{array}$} & & 49,5 & 86,5 \\
\hline & & 47,5 & 85,2 \\
\hline & $N G+V E(n=119)$ & & \\
\hline & $\mathrm{P}_{4}+\mathrm{BE}(\mathrm{n}=122)$ & & \\
\hline
\end{tabular}

eficiente para promover a sincronização da ovulação para todos os tratamentos empregados.

Houve efeito do número de parições (primíparas ou multíparas) nas TP IATF $(\mathrm{P}<0,01)$ e nas TP EM $(\mathrm{P}<0,01)$ conforme verificado na tabela 1 . A TP IATF foi de 35\% para primíparas e $52,7 \%$ para multíparas. A TP EM foi de 71,9\% e 90,2\% para primíparas e multíparas, respectivamente. Observou-se que em fêmeas primíparas as taxas de prenhez foi menor durante toda a estação de monta quando comparadas as multíparas. As TP IATF de 35\% verificada no presente experimento foi um pouco menor do que a $41 \%$ obtida por Soto et $\mathrm{al}^{25}$, entretanto esses autores administraram gonadotrofina coriônica equina (eCG) no dia da retirada dos implantes. Segundo Rossa ${ }^{22}$ o eCG atua promovendo o desenvolvimento folicular final, evento que favorece as taxas de prenhez. Em primíparas um menor desempenho reprodutivo é esperado comparado as multíparas, tanto em rebanhos experimentais como em rebanhos comerciais de cria $^{26,27}$. Deve-se considerar que vacas primíparas ainda não completaram sua fase de crescimento e devem disponibilizar energia para a amamentação. Além disso, primíparas compõem uma classe hierarquicamente inferior quando agrupadas com as multíparas, fato que gera dificuldades no acesso às melhores pastagens, cochos com suplementação mineral e água. De fato, no presente estudo, verificou-se uma interação entre o número de parições (primíparas ou 
Tabela 1 - Taxas de prenhez na inseminação artificial em tempo fixo (TPIATF) e ao término da estação de monta de 49 dias (TP EM) em vacas Nelore, amamentando, tratadas com implantes silásticos, novos ou reutilizados, contendo $3 \mathrm{mg}$ de norgestomet $\left(\mathrm{Crestar}^{\circledR}\right)$ associados a administração de $3 \mathrm{mg}$ de norgestomet (NG) e $5 \mathrm{mg}$ de valerato de estradiol (VE) ou 50mg de progesterona $\left(\mathrm{P}_{4}\right)$ e $2 \mathrm{mg}$ de benzoato de estradiol (BE) no dia da inserção do implante. Todas as vacas receberam na remoção do implante uma injeção de um análogo da PGF2? associada a $1 \mathrm{mg}$ de BE 24 horas após e foram inseminadas 54 a 56 horas depois da retirada do implante

\section{CAUSAS DE VARIAÇÃO}

\begin{tabular}{|c|c|c|}
\hline & TP IATF & TP EM \\
\hline Número de Parições (primíparas ou multíparas) & 0.0095 & 0.0029 \\
\hline Condição Corporal & 0.2996 & 0.6046 \\
\hline Condição Ovariana & 0.7871 & 0.3337 \\
\hline Período Pós Parto & 0.0483 & 0.1604 \\
\hline Característica do implante & 0.5173 & 0.8417 \\
\hline Tratamento administrado no dia da colocação do implante (TAI) & 0.8723 & 0.6110 \\
\hline Número de Parições (primíparas ou multíparas) x Condição Corporal & 0.1044 & 0.0356 \\
\hline Número de Parições (primíparas ou multíparas) x Condição Ovariana & 0.4276 & 0.0822 \\
\hline Número de Parições (primíparas ou multíparas) x Período Pós Parto & 0.1021 & 0.0601 \\
\hline Condição Corporal x Condição Ovariana & 0.3324 & 0.3814 \\
\hline Condição Corporal x Período Pós patto & 0.1143 & 0.3400 \\
\hline Condição Corporal x Característica do implante & 0.3448 & 0.5123 \\
\hline Condição Corporal x TAI & 0.9288 & 0.6951 \\
\hline Condição Ovariana x Período Pós Parto & 0.7593 & 0.9907 \\
\hline Condição Ovariana x Característica do implante & 0.7744 & 0.3087 \\
\hline Condição Ovariana x TAI & 0.4523 & 0.7583 \\
\hline Período Pós Parto x Característica do implante & 0.5822 & 0.7998 \\
\hline Período Pós Parto x TAI & 0.4615 & 0.9465 \\
\hline Característica do implante x TAI & 0.1677 & 0.9755 \\
\hline Número de Parições (primíparas ou multíparas) x Característica do & 0.3146 & 0.5662 \\
\hline implante & 0.8957 & 0.8287 \\
\hline Número de Parições (primíparas ou multíparas) x TAI & 0.4175 & 0.1028 \\
\hline Período Pós Parto x Característica do implantex TAI & 0.0315 & 0.7470 \\
\hline Número de Parições (primíparas ou multíparas)x Característica do & 0.5260 & 0.0928 \\
\hline implante $\times$ TAI & 0.9854 & 0.0866 \\
\hline \multicolumn{3}{|l|}{ Condição Corporal x Característica do implantex TAI } \\
\hline Condição Ovariana x Característica do implantex TAI & & \\
\hline
\end{tabular}

multíparas) e a condição corporal $(\mathrm{P}<0,05)$. Sugere-se que primíparas são mais sensíveis que as multíparas ao efeito do balanço energético negativo no período pós parto. Deve-se considerar que o emprego de programas de IATF em primíparas deve ser direcionado aos animais com condição corporal $\geq 5$, caso contrário o sucesso do emprego de tais programas será comprometido.

No presente estudo, confirma-se a hipótese que não há diferença nas taxas de prenhez entre o emprego de implantes auriculares novos e reutilizados, entretanto, nega-se a hipótese de que em vacas tratadas com $\mathrm{P}_{4}+\mathrm{BE}$ as taxas de prenhez são maiores do que em fêmeas tratadas com $\mathrm{N}+\mathrm{VE}$. Conclui-se que implantes de norgestomet novos e reutilizados, quando associados ao BE ou ao VE promovem taxas de prenhez (TP IATF e TP EM) bastante satisfatórias em vacas Nelore multíparas amamentando.

\section{Agradecimentos}

À Agropecuária J. GALERA pela colaboração na execução deste trabalho. 


\title{
Evaluation of norgestomet auricular implants reused associated to valerate or to estradiol benzoate in fixed time artifical insemination in Nelore cows
}

\begin{abstract}
The use of fixed-time artificial insemination (IATF) in cows is possible by the use of drugs that aim at the synchronization of the folicular wave pool, the estrus and ovulation. In trading cattle the costs of these drugs must stablish a profitable relation with the benefits. The present study meant to compare the pregnancy rates in Nelore (Bos taurus indicus) cows inseminated at fixed-time, treated with new (IN) or once used (IR; reused) auricular releasing devices with $3 \mathrm{mg}$ of norgestomet $\left(\right.$ Crestar $\left.^{\circledR}\right)$, associated with the administration of norgestomet $(\mathrm{NG})$ and estradiol valerate $(\mathrm{VE})$ or progesterone $\left(\mathrm{P}_{4}\right)$ and estradiol benzoate (BE). Pure breed Nelore cows ( $\mathrm{n}=241)$, on lactation with calf received one of the four treatments: new Crestar ${ }^{\circledR}$ during 10 days administrated with $3 \mathrm{mg}$ of $\mathrm{NG}$ and $5 \mathrm{mg}$ of $\mathrm{VE}$ (group IN/NG+VE; $\mathrm{n}=61$ ); new Crestar $^{\circledR}$ inserted during 8 days with $50 \mathrm{mg}$ of $\mathrm{P}_{4}$ and $2 \mathrm{mg}$ of $\mathrm{BE}$ (group IN/P $+\mathrm{BE}$; $\mathrm{n}=61$ ); reused Crestar $^{\circledR}$ inserted during 10 days in association with $3 \mathrm{mg}$ of norgestomet and $5 \mathrm{mg}$ of VE (group IR/NG+VE; $\mathrm{n}=58$ ) or reused Crestar ${ }^{\circledR}$ inserted during 8 days in association with $50 \mathrm{mg}$ of $\mathrm{P}_{4}$ and $2 \mathrm{mg}$ of $\mathrm{BE}$ (group $\mathrm{IR} / \mathrm{P}_{4}+\mathrm{BE} ; \mathrm{n}=61$ ). On the day the releasing devices were removed the animals received 7,5mg of Luprostiol and after 24 hours $1 \mathrm{mg}$ of BE. A fixed-time artificial insemination was done 54 to 56 hours after the removal of the releasing devices. Cows detected in estrus after the insemination at fixed-time were observed during a period of 49 days and reinseminated. The pregnancy diagnosis was done by ultrassonography 35 days after the IATF and after the end of the breeding season. The pregnancy rates of IATF (TP IATF) and at the end of the breeding season (TP EM) were estimated. There was no interaction between the characteristics of the (new or reused) releasing devices and the treatment given at the day the releasing devices were inserted $\left(\mathrm{NG}+\mathrm{VE}\right.$ e $\left.\mathrm{P}_{4}+\mathrm{BE}\right)$. The use of new or used $\mathrm{CIDR}^{\circledR}$ had no effect on TP IATF $(48,3 \%$ vs $48,7 \%)$ and on TP EM $(85,2 \%$ vs $86,5 \%)$. The treatments with $\mathrm{NG}+\mathrm{VE}$ e $\mathrm{P}_{4}+\mathrm{BE}$ did not have effect on TP IATF (49,5\% vs 47,5\%) and TP EM ( 86,5 vs 85,2\%). There was a effect on the number of parturition (primipara and multipara) on the TP IATF (35\% vs $52,7 \%$; P $<0,01)$ and on TP EM $(71,9 \%$ vs $90,2 \% ; \mathrm{P}<0,01)$. It can be concluded that the new or once used releasing devices with norgestomet when associated with $\mathrm{NG}+\mathrm{VE}$ or $\mathrm{P}_{4}+\mathrm{BE}$ promote highly satisfatory pregnancy rates in Nelore cows, being the better rates obtained in multipara females.
\end{abstract}

\section{Referências}

1 ELEY, D. S.; THATCHER, W. W.; COLLIER, R. J.; WILCOX, C.J.; CALL, E.P. Periparturient and postpartum endocrine changes of conceptus and maternal units in Jersey cows bred for milk yield. Journal of Dairy Science, v. 64, p. 312-20, 1981.

2 BRIDGES, P. J.; LEWIS, P. E; WAGNER, W. R.;
Key-words:

Progestens.

Reused.

Estradiol valerato.

Estradiol benzoate.

Bovine 
on follicular development in postpartum beef cows. Journal of Animal Science, v. 64, p. 104-112, 1987.

5 KINDER, J. E.; KOJIMA, F. N.; BERGFELD, E. G. M.; WEHRMAN, M. E.; FIKE, K. E. Progestin and estrogen regulation of pulsatile $\mathrm{LH}$ release and development of persistent ovarian follicle in cattle. Journal of Amimal Science, v. 74, p. 1424-40, 1996.

6 MACMILLAN, K. L. Pharmacological control of the oestrus cycle to improve the reproductive performance of cattle. Revista Brasileira de Reprodução Animal, v. 23 , n. 2 , p. $61-4,1999$

7 BARUFI, F. B. Sincronização do ciclo estral e da ovulação em bovinos de corte usando tratamentos com Crestar, CIDR ou CIDR reutilizado, seguido ou não pela administração de eCG. 2002. 68 p. Dissertação (Mestrado)-Faculdade de Medicina Veterinária e Zootecnia, Universidade de São Paulo, São Paulo, 2002.

8 BARUFI, F. B. et al. Avaliação do uso do Crestar ou CIDR-B + benzoato de estradiol, seguido ou não pela aplicação da gonadotrofina coriônica eqüina (eCG), no desempenho reprodutivo de vacas de corte com bezerros ao pé. Revista da Sociedade Brasileira de Reprodução Animal, v. 23, n. 3, p. 332-333, 1999.

9 BO, G. A. et al. The use of estradiol-17b and progestogen treatment for the control of follicular wave dynamics in beef cattle. Theriogenology, v. 40, p. 65, 1994.

10 MORENO, D. et al. Follicle wave emergency in beef cows treated with progesterone releasing devices, estradiol benzoate and progesterone. Therio-genology, v. 55, p. 408, 2001.

11 VYNCKIER, L. et al. Plasma estradiol-17b concentration in the cow during induced estrus and after injection of estradiol-17b benzoate and estradiol$17 \mathrm{~b}$ cypionate - a preliminary study. Journal of Pharmacology Ther., v. 3, n. 1, p. 36-42, 1990.

12 WILLIANS, C. L; STANCEL, G. C. Estrogênios e progestogênios. In: GOODMAN, H; GILMAN, T. L. As bases farmacológicas da terapeutica. Rio de Janeiro: Mc Graw - Hill, 1996. v. 8, p. 1045-67.

13 ODDE, K. G. A review of syncronization of estrus in postpartum cattle. Journal of Animal Science, v. 68 p.817-30, 1990

14 MACMILLAN, K. L.; PETERSON, A. J. A new intravaginal progesterone relesing devices for cattle CIDR-B) for oestrus syncronization, increasing pregnancy rates and the treatment of pos-partum anoestrus. Animal Reproduction Science, v. 33, n. 1, p. $1-25,1993$

15 MADUREIRA, E. H. Controle farmacológico do ciclo estral com o emprego de progesterona e progestágenos em bovinos. In: Simpósio sobre Controle Farmacológico do Ciclo Estral em Ruminantes, 2000, São Paulo. Anais. São Paulo: Fundação da Faculdade de Medicina Veterinária e Zootecnia, USP, 2000. p. 89-98.

16 COLAZO, N. G. et al. Fixed-time artificial insemination in beef cattle using CIDR-B devices, progesterone and estradiol benzoate. Theriogenology, v. 51, n. 1 , p. 404,1999

17 MARTINEZ, M. F. et al. The use of CIDR-B devices in $\mathrm{GnRH} / \mathrm{LH}$-based artificial insemination programs. Theriogenology, v. 53, n. 1, v. 202-8, 2000.

18 KESLER, D. J. et $\mathrm{al}^{22}$. Comparison of hydron and silicone implants in the bovine norgestomet and estradiol valerate estrus syncronization procedure. Drug Development and Industrial Pharmacy, v. 21, n. 4, p. 475-485, 1995.

19 MACHADO ,JR.; KESLER, D. J. Efficacy of norethindrone acetate and norgestomet implants in suppessing estrus in female beef cattle. Drug Development and Industria e Pharmacy, v. 22, p. 12111216, 1996.

20 Statistical Analisys System. SAS user's guide: statistics. Versão 5. ed. Cary: Statistical Analysis System Institute, 1995.

21 BO, G. A.; ADAMS, G. P.; PIERSON, R. A.; MAPLETOFT, R. J. Exogenous control of follicular wave emergence in cattle. Theriogenology, v. 43, n.1, p. 3140, 1995.

22 ROSSA, L. A. F. Sincronização da ovulação por eCG ou benzoato de estradiol em vacas de corte tratadas com Crestar no período pós parto. 2002. $80 \mathrm{f}$. Dissertação (Mestrado). Faculdade de Medicina Veterinária e Zootecnia, Universidade de São Paulo, São Paulo, 2002.

23 MCGUIRE, W. J. LARSON, R. L.; KIRACOFE, G. H. Syncro-mate B induces estrus in ovariectomized cows and heifers, Theriogenology, v. 34, p. 33-37, 1990.

24 BURKE, C. R. et al. Use of a small dose of estradiol benzoate during diestrus to synchronize development of the ovulatory follicle in cattle. Journal of Animal Science, v. 78, p. 145-151, 2000.

25 SOTO BELLOSO, E. et al. Improvement of reproductive performace in crossbred zebu anestrus primiparous cows by treatment with norgestomet implants or $96 \mathrm{~h}$ calf removal. Theriogenology, v. 57 , p. 1503-10, 2002.

26 WILTBANK, J. N. Research needs in beef cattle reproduction. Journal of Animal Science, v. 31, p. 755, 1970.

27 BELLOWS, R. A.; SHORT, R. E. Effects of precalving feed level on birth weight, calving difficult ans subquesequent fertility. Journal of Animal Science, v. 46, p. 1522,1978

28 WILTBANK, M. C.; GÜMEN, A.; SARTORI, R. Physiological classification on anovulatory conditions in cattle. , v. 57, p. 21-52, 2002. 